\title{
Initial management of potential occult scaphoid fracture in Australasia
}

\author{
Anne-Maree Kelly
}

Received: 18 February 2009 / Accepted: 4 November 2009/Published online: 4 February 2010

(C) Springer-Verlag London Ltd 2010

\begin{abstract}
Aim To characterise current management of adult patients with possible occult scaphoid fracture in Australasian emergency departments.

Methods Internet-based survey of Directors of Emergency Medicine Training throughout Australasia. Data collected included the most common management used in ED for patients with possible occult scaphoid fracture and whether there was a guideline regarding management of such cases. Data are reported as descriptive statistics.

Results 61 responses were received (response rate $73 \%$ ). The most common management reported was immobilisation in a backslab $(23,38 \%)$ or full cast $(19,32 \%)$ with clinical assessment and re-X-ray in 7-10 days. CT scan within 7 days was used by $9(15 \%)$, bone scan within 7 days by $6(10 \%)$ and MRI within 7 days by $3(5 \%)$. Very few sites were using same day/next day CT or MRI. Eightythree percent of sites reported not having a guideline/ protocol for this condition.

Conclusion The traditional approach to management of possible occult scaphoid fracture of immobilisation with re$\mathrm{X}$-ray at 7-10 days remains the most commonly used in Australasia, despite evidence that this is probably overtreatment with significant consequences for patients. The place of advanced imaging for investigation of potential scaphoid fractures requires further research.
\end{abstract}

Keywords Scaphoid $\cdot$ Fracture $\cdot$ Management $\cdot$ Imaging

A.-M. Kelly $(\bowtie)$

Joseph Epstein Centre for Emergency Medicine Research,

Western Health,

Melbourne, Australia

e-mail: anne-maree.kelly@wh.org.au

\section{Introduction}

Management of suspected scaphoid fractures with normal $\mathrm{X}$-rays at emergency department (ED) presentation is a common clinical challenge. There has long been concern that failure to identify and immobilise a scaphoid fracture might result in avascular necrosis or non-union with significant functional impact for the patient and potential medico-legal risk for the practitioner. Traditional management would see the patient immobilised in a plaster cast for 7-14 days followed by clinical examination and re-X-ray to identify fractures not visible on the initial films [1]. That approach can been challenged on two fronts: whether cast immobilisation while awaiting clinical review is justified and the role of alternative imaging modalities at or near the time of initial presentation. Little is known about current practice patterns across Australasian ED for this condition. The aim of this project was to characterise current management of adult patients with possible occult scaphoid fracture in Australasia.

\section{Methods}

This was an Internet-based survey of Directors of Emergency Medicine Training (DEMT) throughout Australasia as identified from the Australasian College for Emergency Medicine web-site (www.acem.org.au). DEMTs are the designated supervisors of specialist training at hospitals accredited for training, both metropolitan and rural (one/ hospital). As such, they are easily identifiable, experienced specialists with a good understanding of clinical practice within the ED in which they work. Participants were sent an invitation to participate in the confidential on-line survey by 
e-mail (where one could be found) or by post. A minimum of two reminders were sent to maximise participation.

Data were collected using Internet-based survey software (www.surveymonkey.com) and included the most common management strategy used for patients with possible occult scaphoid fracture (from a range of options, see Table 1) and whether there was a written ED guideline regarding management of such cases. The outcome of interest was the most common management strategy for possible occult scaphoid fractures. Data are reported as descriptive statistics.

This project was approved by Western Health under the NHRMC Quality Assurance Guidelines.

\section{Results}

61 responses were received, representing a response rate of $73 \%$. The most common management reported was immobilisation in a backslab $(23,38 \%)$ or full cast (19, $32 \%$ ) with clinical assessment and re-X-ray in 7-10 days. CT scan within 7 days was used by $9(15 \%)$, bone scan within 7 days by $6(10 \%)$ and MRI within 7 days by 3 (5\%). One ED reported same/next day CT (Table 1).

Eighty-three percent of sites reported not having a written guideline/protocol for this condition.

\section{Discussion}

The rate of occult scaphoid fractures in cohorts of patients with clinical evidence suggestive of scaphoid fracture but normal initial X-rays is reported to be $0-16 \%$ [2-4], weighted average $3.7 \%(95 \%$ CI $2.5-5.6 \%)$. In the subgroup who prove to have a fracture, the rate of delayed/non-union is low (10-20\%) [2]. Nonetheless, nonunion or avascular necrosis can result in significant morbidity. Traditional treatment for suspected occult scaphoid fracture is cast immobilisation with clinical reassessment and re-X-ray at 7-10 days [1]. That approach, however, has been suggested to be over-treatment, with a very low rate of significant fractures at the cost of significant time lost from work or school [5-7]. It has been suggested that symptomatic treatment with follow-up within 2 weeks for these cases is sufficient $[6,8,9]$.

We found that most Australasian EDs continue to use plaster immobilisation for this patient group (backslab or full cast, $70 \%$ ) and that uptake of alternative imaging modalities is only moderate (30\%). Very few EDs are using same day/ next day imaging for this group. The high use of immobilisation is at odds with reported UK practice, where only $46 \%$ of EDs reported routinely using plaster immobilisation for X-ray-negative suspected scaphoid fracture [10].

A few clinical studies have compared management strategies for this patent cohort. CT, MRI and bone scan have all been shown to be effective in the diagnosis of occult scaphoid fractures [11], but to date no studies have shown additional benefit from early advanced imaging for hard clinical outcomes. At present, the logical role for advanced imaging is for patients with persistent symptoms and normal follow-up X-rays. There is a clear need for further research comparing combinations of the various immobilisation and imaging strategies and reporting clinical outcomes (including time off work and time immobilised) and cost-effectiveness

Table 1 Management options and reported frequency

\begin{tabular}{lc}
\hline Management strategy & Frequency (N, \%) \\
\hline Backslab (half-cast) with assessment and re-X-ray at $7-14$ days & $23,38 \%$ \\
Plaster cast (full) with assessment and re-X-ray at $7-14$ days & $19,32 \%$ \\
Backslab with early CT (within 7 days) & $6,10 \%$ \\
Backslab with early bone scan (within 7 days) & $5,8 \%$ \\
Backslab with early MRI (within 7 days) & $1,3 \%$ \\
Support bandage with early MRI (within 7 days) & $1,7 \%$ \\
Support bandage with early CT (within 7 days) & $1,7 \%$ \\
Plaster cast (full) with early CT (within 7 days) & $1,1.7 \%$ \\
Plaster cast (full) with early bone scan (within 7 days) & $1,1.7 \%$ \\
Support bandage with re-assessment at 1 week, with further tests only if symptoms or signs persist & 0 \\
Support bandage with assessment and re-X-ray at $7-14$ days & 0 \\
Plaster cast (full) with early MRI (within 7 days) & 0 \\
Support bandage with early bone scan (within 7 days) & $1,1.7 \%$ \\
Other & \\
Same/next day CT & \\
\hline
\end{tabular}


outcomes. There is no evidence that lack of access to advanced imaging impacts adversely on clinical outcome.

The reasons for the persistent use of plaster immobilisation in Australian ED is unclear. The weight of evidence would suggest that this is over-treatment and that a simple bandage is sufficient. The likely explanations are habit and fear of medicolegal risk.

This study has some limitations that should be considered when interpreting the results. As this was a survey, the management strategy reported may not be that actually used. Only one clinician per ED was surveyed. This would not identify within-ED variations in practice.

\section{Conclusion}

The traditional approach to management of possible occult scaphoid fracture of immobilisation with re-X-ray at 7 10 days remains the most commonly used in Australasia, despite evidence that this is probably over-treatment with significant consequences for patients. The place of advanced imaging for investigation of potential scaphoid fractures requires further research.

\section{Conflicts of interest None.}

\section{References}

1. McRae R (1981) Practical fracture treatment. Churchill Livingstone, New York

2. Best BETS. Immobilisation of suspected scaphoid fractures. 2000. http://www.bestbets.org/bets/bet.php?id=97. Accessed Oct 2008

3. Pillai A, Jain M (2005) Management of clinical fractures of the scaphoid: results of an audit and literature review. Eur J Emerg Med 12:47-51

4. Mittal RL, Dargan SK (1989) Occult scaphoid fracture: a diagnostic enigma. J Orthop Traumatol 3:306-308

5. Duncan DS, Thurston AJ (1985) Clinical fracture of the carpal scaphoid-an illusionary diagnosis. J Hand Surg [Br] 10:375-376

6. Jacobsen S, Hassani G, Hansen D et al (1995) Suspected scaphoid fractures. Can we avoid overkill? Acta Orthop Belg 61:74-78

7. Sjolin SU, Andersen JC (1988) Clinical fracture of the carpal scaphoid-supportive bandage or plaster cast immobilization? J Hand Surg [Br] 13:75-76

8. McLaughlin H, Parkes JC (1969) Fracture of the carpal navicular (scaphoid) bone: gradations in therapy based upon pathology. J Trauma 9:311-319

9. Zarnett R, Martin C, Barrington TW et al (1991) The natural history of suspected scaphoid fractures. Can J Surg 34:334 337

10. Tai CC, Ramachandran M, McDermott ID et al (2005) Management of suspected scaphoid fractures in accident and emergency departments-time for new guidelines. Ann R Coll Surg Engl $87: 353-357$

11. Blum A, Sauer B, Detreille R et al (2007) The diagnosis of recent scaphoid fractures: review of the literature. J Radiol 88:741-759 